\title{
Medicina traslacional: un puente de plata entre las ciencias básicas y la medicina clínica
}

\author{
Translational medicine: a silver bridge between basic sciences and clinical medicine
}

El término 'Medicina traslacional'es un anglicismo que inicialmente implicó el concepto de 'trasladar y aplicar los hallazgos obtenidos en el mesón de laboratorio a la cama del paciente’. O sea, en términos generales el objetivo de la medicina traslacional es facilitar la transición de la investigación básica en aplicaciones clínicas que redunden en beneficio de la salud'.

Inicialmente la medicina traslacional fue descrita como "el matrimonio entre nuevos hallazgos en ciencia básica y la práctica clínica"'. En la última década la medicina traslacional se ha constituido en una disciplina emergente y progresivamente vigorosa especialmente en los centros académicos dedicados a cultivar las ciencias médicas. Sin embargo, estos avances han tenido lugar sin una clara definición de la disciplina. Es así como el término puede ser interpretado en forma diferente según el campo de acción del investigador. Para los investigadores en ciencias básicas y preclínicas realizar medicina traslacional es traducir el conocimiento sobre nuevos mecanismos y técnicas en nuevos enfoques de diagnóstico y tratamiento de determinadas enfermedades. En tanto que para los investigadores en salud pública la medicina traslacional significa asegurar que los hallazgos sobre prevención, diagnóstico o tratamiento sean aplicados apropiadamente a la población ${ }^{3}$.

La producción de un nuevo fármaco que sería el punto final para un investigador básico o preclínico, pasa a ser el punto de partida para el investigador en salud pública y los servicios de salud, cuyo propósito es mejorar la calidad de acceso, organización y coordinación de los sistemas de cuidadol.

Aunque los desafios enfrentados por ambos enfoques son muy disimiles, la mayoría de las personas tienen en mente el enfoque del investigador básico o preclínico cuando se usa el término de medicina traslacional. Sin embargo, se debe considerar que la investigación traslacional en medicina requiere de la interacción de numerosos protagonistas que plantearán una interrogante desde la cama del paciente, la llevarán a modelos experimentales desarrollados en el laboratorio para generar una eventual respuesta a la pregunta planteada, la cual finalmente deberá ser resuelta aplicando la solución encontrada al paciente y eventualmente a la población ${ }^{3}$.

La existencia de varias definiciones de medicina traslacional refleja la diversidad institucional en investigación traslacional y sus programas de desarrollo.

El editor del Journal of Translational Medicine en el primer número de esta revista publicado en el año 2003, manifiesta que el propósito de la investigación traslacional en medicina es probar en seres humanos nuevas estrategias terapéuticas desarrolladas a través de la experimentación y plantea que la investigación traslacional debe ser considerada como un camino bidireccional: "desde el mesón del laboratorio a la cama del paciente y viceversa". Reconoce que los esfuerzos en la dirección "cama del paciente $\rightarrow$ mesón del laboratorio" han estado limitados porque los tópicos científicos son comprendidos insuficientemente por los clínicos a tiempo completo y porque a su vez los investigadores en ciencias básicas no aprecian en su total complejidad las dificultades que se presentan al tratar seres humanos enfermos. En este escenario el Journal of Translational Medicine declara promover la publicación expedita de resultados obtenidos a través de investigaciones en seres humanos que pueden beneficiar a la comunidad cientifica y viceversa. Una caracteristica relevante de esta revista es que su comité editorial está constituido por especialistas con conocimientos especializados tanto en clínica como en las ciencias básicas ${ }^{4}$.

Por su parte en 2012, la editorial del número inaugural de la revista Clinical and Translational Medicine 
define el término Medicina Clínica y Traslacional como "el potencial uso clínico y la aplicación de la investigación traslacional para mejorar la comprensión del mecanismo y tratamiento de las enfermedades humanas", lo cual facilitaría el desarrollo de biomarcadores especificos de enfermedad y estrategias terapéuticas para el seguimiento y curación de enfermedades ${ }^{5}$. Además plantea que la medicina traslacional juega un papel singular y crucial en fomentar el flujo de información bidireccional entre investigadores básicos y clínicos, optimizando nuevas biotecnologías, mejorando la aplicación de nuevos conceptos terapéuticos y en último término mejorando la calidad de vida de los pacientes.

La European Society for Translational Medicine (EUSTM) en su declaración de intenciones ha definido la medicina traslacional como "una rama interdisciplinaria del área biomédica sustentada por tres pilares principales: el trabajo de laboratorio (benchside), el trabajo clínico (bedside) y la comunidad. Según la EUSTM el propósito de la medicina traslacional es combinar disciplinas, recursos, conocimientos y técnicas, para promover mejorías en prevención, diagnóstico y tratamiento de las enfermedades". Esta amplia definición considera a la medicina traslacional como un área altamente interdisciplinaria cuya meta fundamental es amalgamar habilidades de diversa naturaleza con el propósito de mejorar los sistemas de atención de salud de la humanidad ${ }^{6}$.

Entre los impactos positivos que puede producir la medicina traslacional se han señalado los siguientes ${ }^{5}$ : a) integración de la investigación clínica con metodologías de punta tales como biología computacional, imagenología avanzada y metodología de las así llamadas ciencias ómicas ${ }^{7}$ como la genómica que estudia el contenido, la organización, la función y la evolución de la información molecular del ADN contenido en el genoma de los seres vivos, la trascriptómica que estudia los perfiles de expresión de los genes presentes en el genoma a través del estudio del conjunto de ARN que existe en una célula, tejido u órgano, la proteómica que estudia la estructura y función de las proteínas, la metabolómica que estudia los metabolitos celulares de bajo peso molecular en muestras biológicas y la farmacogenómica que estudia el conjunto de genes farmacológicamente relevantes ${ }^{7}$; b) implementación de bancos de tejido humano y desarrollo de bio-bancos ligados a bases de datos clínicos de alta calidad para la identificación de fenotipos relevantes de pacientes portadores de enfermedades especificas; c) validación de la aplicación de nuevas tecnologías en determinados enfermos; d) desarrollo de nuevas estrategias para mejorar la calidad de vida en determinados pacientes; e) agrega evidencia cientifica a normas, a políticas de salud pública y/o a guías para el uso de medicamentos, terapias, enfoques preventivos y entrega de atención sanitaria.

A pesar de todos estos impactos positivos la medicina traslacional ha sido criticada por su alto costo ya que instituciones privadas han llegado a invertir hasta un billón de dólares en el desarrollo de un solo medicamento, lo cual implicaría un negocio de inversión con elevadas ganancias a largo plazo. Esta situación motivó que las agencias reguladoras de la investigación del Reino Unido en 2007 y de EE.UU. en 2010 instauraran regulaciones legislativas en relación a la medicina traslacional ${ }^{8}$.

PubMed registraba desde 1988 hasta el 28 de julio de 2017, 85.224 publicaciones catalogadas en el item translational medicine correspondiendo $2.058(2,1 \%)$ de esas publicaciones al item translational respiratory medicine (Medicina Respiratoria Translacional).

Si bien la denominación de Medicina Traslacional y las revistas y sociedades dedicadas a esta disciplina son de reciente creación, la idea de aplicar conocimientos adquiridos en los laboratorios experimentales a condiciones clínicas es de muy larga data y tal vez una aspiración fundacional de la medicina experimental concebida en la época de Claude Bernard (1813-1878). La historia de la medicina es muy generosa en ejemplos de aplicación de conocimientos básicos a la solución de problemas clínicos y en Medicina respiratoria un caso paradigmático es el del surfactante pulmonar ${ }^{9,10}$ vislumbrado en 1929 por Kurt von Neergard, fue redescubierto 26 años después por John Clements en EE.UU y Richard Pattle en Inglaterra, ulteriormente Mary Ellen Avery \& Jere Mead en 1959 comunicaron que su déficit producia la membrana hialina del recién nacido, enfermedad que se pudo prevenir con la administración de glucocorticoides a la madre 48 h antes del parto y más aún Fujiwara y cols en 1980 trataron exitosamente a recién nacidos con membrana hialina instilándoles intratraquealmente surfactante exógeno y actualmente existen preparados de surfactante disponibles en la farmacopea y en los Programas Nacionales de Surfactante que han disminuido significativamente la mortalidad de los prematuros en países desarrollados $y$ también en Chile ${ }^{10}$. Parecería entonces que Medicina Traslacional es un nuevo nombre para una práctica anti- 
gua en la medicina. Sin embargo, debemos reconocer que el concepto de Medicina Traslacional está muy ligado al desarrollo de la Biología molecular y a sus nuevas tecnologías cuyo desarrollo exponencial fue creando una brecha insalvable entre los médicos clínicos y quienes se dedican a las ciencias básicas. El sistema de investigaciones biomédicas y la práctica clínica evolucionaron durante años por vías divergentes con lo cual la interacción bidireccional "cama del paciente $\leftrightarrow$ mesón del laboratorio" fue sufriendo un progresivo y profundo deterioro. Los paises desarrollados encabezados por EE. UU. y el Reino Unido trataron de superar esta brecha traslacional fomentando este tipo de investigación destinándole recursos financieros, materiales y humanos ${ }^{1,3,11}$. Los National Institutes of Health (NIH) de EE.UU han creado 60 centros de Ciencia y Clinica Traslacional, se trata de centros multidisciplinarios que incluyen científicos (Ph.Ds), médicos, bioinformáticos, estadísticos, ingenieros y expertos en industrias, ya que actualmente es imposible hacer medicina traslacional en solitario. En Europa existen más de 20 agencias que están siguiendo el ejemplo de los $\mathrm{NIH}^{1}$.

En América Latina los paises que lideran la productividad de la región en investigación biomédica están realizando programas de fortalecimiento de los proyectos en medicina traslacional. Nuestro país no ha estado ajeno a estas iniciativas, a pesar de los habitualmente escasos recursos dispuestos por el Estado y la industria privada nacional a la investigación cientifica, se han formado centros y laboratorios dedicados a la medicina traslacional, como por ejemplo el Laboratorio de Inmunología Traslacional de la Facultad de Medicina de la P. Universidad Católica ${ }^{12}$ y el Centro de Investigación Clínica Avanzada (CICA) creado en 2016 en el Hospital Clínico de la Universidad de Chile en base a médicos clínicos y a médicos egresados del programa de doctorado en Ciencias Médicas de la Facultad de Medicina, Universidad de Chile ${ }^{13}$.

Todos estos esfuerzos globales por mejorar la integración de la investigación básica y clínica no deberian descuidar el fomento de la ciencia básica pilar fundamental del conocimiento científico. A nivel nacional la vieja disyuntiva de fomentar la ciencia básica o la aplicada debería dirimirse en favorecer a la buena ciencia independientemente de otros calificativos, no olvidando el "factor humano" que apunta a la promoción de investigadores jóvenes talentosos, compasivos e inspirados por buscar la respuesta a sus preguntas dirigidas a develar los secretos propios de la naturaleza o de las enfermedades.

Dr. Manuel Oyarzún Gómez

Profesor titular, Programa de Fisiopatología

Instituto de Ciencias Biomédicas

Facultad de Medicina, Universidad de Chile Miembro de número Academia Chilena de Medicina

Email:moyarzun@med.uchile.cl

\section{Bibliografía}

1. BECÚ-VILLALOBOS D. Medicina traslacional, ¿moda o necesidad? Medicina (B. Aires) 2014; 74: 170-2.

2. GERAGHTY J. Adenomatous poliposis coli and traslational medicine. Lancet 1996; 348 (9025): 422.

3. REY R. Investigación traslacional en medicina. Rev Hosp Niños (B. Aires) 2016; 58: 142-8.

4. MARINCOLA F M. Translational medicine: A to way-road. J Transl Med 2003; 1: 1-2.

5. ABRAHAM E, MARINCOLA F M, CHEN Z, WANG X. Clinical and translational medicine: integrative and practical science. Clinical and Translational Medicine 2012; 1: 1-3.

6. COHRS R J, MARTIN T, GHAHRAMANI P,
BIDAUT L,HIGGINS P J, SHAHAZAD A. Translational medicine definition by the European Society for Translational Medicine. New Horizons in Translational Medicine 2015; 2: 86-8.

7. BERNAL RUIZ M L. La era de las ciencias ómicas. Academia de Farmacia del Reino de Aragón. Zaragoza.17 junio, 2015. Disponible en: http:// www.academiadefarmaciadearagon.es/docs/Documentos/Documento73.pdfn (consultado el 29 de julio de 2017).

8. SHIGUETOMI MEDINA J M. La medicina traslacional: una disciplina emergente. Rev Mex Ortop Ped 2013; 15: 64-7.

9. COMROE Jr J H. Premature science and immature lungs. En: Restrospectroscope. Insights into medical discovery. Von Gehr Press, Merlo Park, California, USA 1977. págs 140-82. 
10. OYARZÚN M. Surfactante pulmonar un modelo precoz de medicina traslacional. Bol Academia Chilena de Medicina 2013; 50: 189-202.

11. ZERHOUNI E A. Translational and clinical science-Time for a new vision. N Engl J Med 2005; 353: 1021-3.

12. BORZUTZKY A. http://medicina.uc.cl/inves- tigadores/arturo-borzutzky. (consultado el 1 de agosto de 2017).

13. VALENZUELA C. Sinergia en la base de la medicina traslacional. Disponible en: http://www. uchile.cl/noticias/134229/sinergia-en-la-base-dela-medicina-traslacional (consultado el 29 de julio de 2017). 